\title{
Desempeño clinimétrico de la Escala de Evaluación de la EPOC (CAT)
}

\author{
John Carlos Pedrozo-Pupo, Adalberto Campo-Arias 凶
}

Facultad de Ciencias de la Salud, Santa Marta, Colombia, Universidad del Magdalena.

Trabajo recibido: 08-V-2017; aceptado: 17-V-2017

\begin{abstract}
RESUMEN. Introducción: La escala de evaluación de la EPOC, en inglés COPD Assessment Test o CAT, es una herramienta para evaluar la severidad de los síntomas en EPOC; sin embargo, se desconoce la confiabilidad y la dimensionalidad en el contexto colombiano. Objetivo: Conocer la confiabilidad y dimensionalidad de la CAT en una muestra de pacientes en la ciudad de Santa Marta, Colombia. Material y método: Se diseñó un estudio clinimétrico o metodológico con la participación de 292 adultos con diagnóstico de EPOC, en edades entre 49 y 95 años (media = 73.5; DE = 8.7), 61.6\% hombres 38.4\% mujeres. Los participantes diligenciaron los ocho incisos de la CAT. Se determinó consistencia interna (alfa de Cronbach y omega de McDonald) y dimensionalidad (análisis factorial exploratorio y confirmatorio, AFE y AFC). Resultados: Se observó consistencia interna de 0.71 tanto para alfa de Cronbach como para omega de McDonald. La solución para la única dimensión explicó el $34.4 \%$ de la varianza, con pobres indicadores de ajustes de bondad en el AFC $\left(\chi^{2}=126.1 ; \mathrm{gl}=20 ; p=0.001 ; \mathrm{RMSEA}=\right.$ 0.13; IC 90\% 0.11-0.15; CFI = 0.75 y TLI = 0.66, y SMSR =0.08). Conclusiones: La CAT presenta aceptable consistencia interna; sin embargo, la estructura unidimensional muestra un pobre ajuste en pacientes con EPOC de Santa Marta, Colombia. Es necesario corroborar estos hallazgos en otras poblaciones.
\end{abstract}

Palabras clave: Enfermedad pulmonar obstructiva crónica, calidad de vida, confiabilidad y validez, estudios de validación.

ABSTRACT. Background: The COPD assessment test (CAT) is a tool to assess the quality of life among patients with chronic obstructive pulmonary disease (COPD). However, its reliability and dimensionality are unknown in the Colombian context. Objective: To know the reliability and dimensional of CAT in a sample of patients from the city of Santa Marta, Colombia. Material and method: A clinimetric or methodological study was designed with participation of 292 adults suffered from COPD, aged between 49 and 95 years (mean $=73.5$, SD $=8.7$ ), 61.6\% of sample were men and $38.4 \%$, women. Participants completed the eight items of CAT. Internal consistency (Cronbach's alpha and McDonald's omega) and dimensionality (exploratory and confirmatory factor analysis, EFA and CFA) were determined. Results: Internal consistency of 0.71 was observed for both Cronbach's alpha and McDonald's omega. The solution for the one dimension explained $34.4 \%$ of the variance, with poor indicators of goodness adjustments in the CFA $\left(\chi^{2}=126.1, \mathrm{df}=20, \mathrm{p}=0.001, \mathrm{RMSEA}=0.13, \mathrm{IC} 90 \% 0.11-0.15, \mathrm{CFI}=0.75, \mathrm{TLI}=0.66\right.$ and SMSR $=0.08$ ). Conclusions: The CAT has modest internal consistency; however, the one-dimensional structure shows poor goodness of fit in patients with COPD in Santa Marta, Colombia. It is necessary to corroborate these findings in other populations.

Key words: Chronic obstructive pulmonary disease, quality of life, reliability and validity, validation studies.

\section{INTRODUCCIÓN}

El desempeño clinimétrico o psicométrico de los instrumentos de medición en salud es característico de cada población, es decir, presenta variaciones importantes y esto puede limitar la utilidad en algunos contextos. ${ }^{1}$

En el año 2009, Jones et al. publicaron la escala de evaluación de la EPOC, en inglés COPD Assessment Test o CAT. Éste es un instrumento de ocho preguntas para evaluar la calidad de vida en este grupo de pacientes y mostró alta consistencia interna, alfa de Cronbach de 0.88 y estructura unidimensional. ${ }^{2}$

Se ha informado un buen desempeño clinimétrico para la CAT en diferentes poblaciones. Por ejemplo,
Gupta et al. realizaron una revisión sistemática de los estudios disponibles desde la introducción del instrumento hasta el año 2013 y hallaron consistencia interna con valores entre 0.85 y 0.98 y aceptables coeficientes en la exploración de la validez (convergente, discriminante y predictiva). ${ }^{3}$ Por otra parte, Weldam et al. revisaron las escalas para evaluar la calidad de vida en pacientes con EPOC e informaron un único estudio que probó la estructura interna unidimensional para la CAT mediante análisis Rasch. ${ }^{4}$

Dado el adecuado desempeño que mostró la CAT en la versión inglesa se realizaron traducciones a otros idiomas. ${ }^{5-14}$ Sin embargo, la consistencia interna y la dimensionalidad de la CAT no han sido evaluadas en 
pacientes de habla hispana. Por una parte, la consistencia interna de la CAT ha sido exageradamente alta en algunas investigaciones, sin que se prestara atención a ello. ${ }^{3-5}$ De la misma forma, no se ha informado la omega de McDonald como una medida más apropiada de la confiabilidad de una escala de medición en salud cuando el valor de los coeficientes para cada ítem en el análisis de factores es lo suficientemente heterogéneo. ${ }^{15}$ Y por otra, no se puede garantizar la dimensionalidad de un instrumento a partir de una única investigación. ${ }^{2,4}$ La estructura interna de los instrumentos de mediciones en salud se deben evaluar repetidamente en diferentes poblaciones. ${ }^{1}$ Sin duda, los hallazgos del presente estudio ayudarán a decidir el uso de la CAT en estudios clínicos y epidemiológicos en lengua castellana.

El objetivo del presente estudio fue conocer la confiabilidad y dimensional de la CAT en una muestra de pacientes en la ciudad de Santa Marta, Colombia.

\section{MATERIAL Y MÉTODO}

La presente es una investigación clinimétrica, es decir, un estudio metodológico o de evaluación de instrumento de tamización, diagnóstico o gradación clínica. Esta investigación contó con la revisión y aprobación de un comité de ética en investigación. Los participantes firmaron consentimiento informado en consonancia con la legislación colombiana para la investigación con personas y la Declaración de Helsinki.

Este estudio se realizó en Santa Marta, Colombia, una ciudad a nivel del mar en la costa Caribe colombiana, con una población aproximada de 450,000 habitantes. Se tomó una muestra consecutiva de 292 pacientes con diagnóstico de EPOC evaluado durante el año 2016 en la Clínica Marcaribe, el Hospital Universitario Fernando Troconis y Respire Instituto para el cuidado respiratorio. Este tamaño de muestra permite satisfactoriamente el cálculo de la consistencia interna y realizar análisis factorial exploratorio (AFE) y confirmatorio (AFC).$^{16}$ Las edades de los participantes se observaron entre 49 y 95 años, con una media de 73.5 años (DE = 8.7). En relación con el sexo, hicieron parte de la investigación 180 hombres (61.6\%) y 112 mujeres (38.4\%).

Los participantes se clasificaron según la guía GOLD (Global Initiative for Chronic Obstructive Lung Diseases) y diligenciaron la CAT. La clasificación GOLD que gradúa la severidad de la EPOC en cuatro categorías de A, B, C y $\mathrm{D}$, a partir de una valoración multidimensional. ${ }^{17}$ Como antes se anotó, este instrumento se compone de ocho preguntas sencillas que se responden entre dos y tres minutos. Esta escala cuantifica la severidad de la tos, la presencia de flemas, la opresión torácica, la falta de aire durante el esfuerzo, la actividad doméstica, la sensación de seguridad al salir del hogar, la calidad del sueño y el nivel de energía. Cada punto brinda seis opciones de respuesta que se califican de cero a cinco, de menor a mayor severidad, por lo tanto las puntuaciones totales se ubican en el rango entre cero y cuarenta. ${ }^{2}$

Al inicio del estudio se llevó a cabo una prueba piloto con diez pacientes para explorar la comprensión de cada una de las preguntas y hacer ajustes en la redacción sin variar sentido lingüístico de ellas. ${ }^{18}$ Los participantes completaron la CAT con la ayuda del familiar acompañante. La autoadministración y la administración como entrevista de esta escala muestran rendimientos clinimétricos similares. ${ }^{19}$

Para conocer la consistencia interna se calcularon los coeficientes de alfa de Cronbach $^{20}$ y omega de McDonald. ${ }^{21} \mathrm{La}$ validez nomológica se estableció con el coeficiente de correlación de Spearman $\left(r_{s}\right)$, que aplica para correlaciones con datos ordinales, como la clasificación GOLD usada en el presente estudio. ${ }^{22}$ Valores de $r_{s}$ iguales o superiores a 0.30 se tomaron como punto de corte para aceptar una buena validez nomológica del instrumento, independientemente del valor de probabilidad. Se realizó una prueba de validez discriminante al comparar (media y desviación estándar) de las puntuaciones en la CAT según los cuatro grupos que determina la guía GOLD (A, B, C y D). Para esto se calculó la homogeneidad de la varianza con la prueba de Levene y la prueba $\mathrm{F}$ de ANOVA. Además se realizaron múltiples comparaciones post hoc con la prueba de Tukey.

Para conocer la posibilidad de realizar AFE y AFC se calculó el coeficiente de esfericidad de Bartlett ${ }^{23}$ y la prueba de adecuación de la muestra de Kaiser Meyer Olkin, KMO. ${ }^{24}$ Estos coeficientes indican la presencia de un factor latente en un conjunto de preguntas. Los análisis factoriales se realizaron con el método de máxima verosimilitud. Se observaron comunalidades y coeficientes en la matriz y la bondad del ajuste en el AFC se analizó con la prueba de la $\chi^{2}$ de Satorra-Bentler, con grados de libertad ( $\mathrm{gl}$ ) y valor de probabilidad (p), el RMSEA (Root Mean Square Error of Approximation, raíz del cuadrado media del error de aproximación) e intervalo de confianza del 90\% (IC 90\%), el CFI (Comparative Fit Index, índice comparativo de ajuste), el índice de Tucker-Lewis (TLI) y el SMSR (Standardized Mean Square Residual, residuo cuadrado promedio estandarizado). Para $\chi^{2}$ de Satorra-Bentler se acepta favorable si el valor de probabilidad es mayor a 5\%, para RMSEA y SMSR si son menores de 0.06 , para CFI y TLI valores son superiores 0.89 y para SMSR si es menor de 0.05 . Estas pruebas se llevaron a cabo con los programas estadísticos SPSS ${ }^{25}$ y STATA. ${ }^{26}$ 


\section{RESULTADOS}

Las puntuaciones en la CAT se observaron entre $0 \mathrm{y}$ 38 , media $=14.4(D E=8.1)$. La consistencia interna fue 0.71 tanto para alfa de Cronbach como para omega de McDonald. Para la validez nomológica estos pacientes, según la clasificación GOLD se distribuyeron así: 28 pacientes (9.6\%) grado $A ; 128$ (43.8\%) grado $B ; 108$ (37.0\%) grado C; y $28(9.6 \%)$ grado D. La correlación entre las puntuaciones en la CAT y la gradación GOLD mostró coeficiente $r_{s}$ de 0.25 .

Para la validez discriminante, se encontró que el grupo GOLD A mostró una media en la puntuación en la CAT de 12.3 (DE = 8.4), el GOLD B de 12.5 (DE = 7.1), el GOLD C de 16.1 (7.8) y el GOLD D de 18.1 (DE = 9.8). La varianza fue homogénea en los cuatro grupos (estadísticos de Levene $=2.2 ; \mathrm{gl}=3, \mathrm{p}=0.08$ ). En general, la ANOVA mostró diferencias significativas entre los grupos $(F=7.7 ; g l=3 ; p<0.001)$. Sin embargo, la prueba de Tukey no mostró diferencias significativas entre los grupos, entre GOLD A, GOLD B y GOLD C con valor de $p=0.07$ y entre GOLD C y GOLD D, el valor de $p=0.43$.

En el análisis factorial se observó un $\chi^{2}$ de 451.3; grados de libertad $=28$ y valor de $p=0.001$ y el coeficiente KMO fue 0.73. El AFE mostró dos dimensiones: la primera con valor propio (Eigen value) de 2.75 que explicó el $34.4 \%$ de la varianza; y la segunda, con valor de propio de 1.44 responsable del $18.0 \%$ de la varianza total. Las comunalidades y matriz de coeficientes se presentan en la tabla 1.

En el AFC los indicadores de ajustes de bondad fueron pobres, tanto para la solución unidimensional $\left(\chi^{2}=126.1 ; \mathrm{gl}=20 ; \mathrm{p}=0.001 ; \mathrm{RMSEA}=0.13 ; \mathrm{IC} 90 \%\right.$ $0.11-0.15 ; \mathrm{CFI}=0.75$ y TLI $=0.66$, y SMSR $=0.08)$,

Tabla 1. Comunalidades y coeficientes para las preguntas del CAT (AFE).

\begin{tabular}{lccc}
\hline & & \multicolumn{2}{c}{ Coeficiente } \\
\cline { 3 - 4 } Ítem & Comunalidad* $^{*}$ & Factor I & Factor II \\
\hline 1. Tos & 0.736 & & 0.858 \\
2. Flema & 0.391 & & 0.625 \\
3. Opresión & 0.244 & 0.479 & \\
4. Falta de aire & 0.487 & 0.697 & \\
5. Actividad & 0.577 & 0.752 & \\
6. Seguridad & 0.256 & 0.506 & \\
7. Sueño & 0.128 & 0.354 & \\
8. Energía & 0.324 & 0.568 & \\
& & &
\end{tabular}

* Una o más comunalidades presentaron comunalidades superiores a 1 cuando se consideraron las interacciones. Esta solución se debe interpretar con mucha precaución. como para la bidimensional $\left(\chi^{2}=37.0 ; \mathrm{gl}=19 ; \mathrm{p}=0.001\right.$; RMSEA = 0.06; IC 90\% 0.03-0,08; CFI = 0.96 y TLI = 0.94, y SMSR $=0.04$ ).

\section{DISCUSIÓN}

El desempeño clinimétrico de los instrumentos de medición en salud es ampliamente variable. ${ }^{1}$ En la presente investigación se observó un aceptable valor de consistencia interna para la CAT, medida con dos coeficientes para afrontar las limitaciones propias del alfa de Cronbach. ${ }^{27}$ Este valor fue muy inferior a lo que se observó en estudios precedentes, con excepción de la versión en gallego que mostró un idéntico coeficiente..$^{2-4,6-9}$ Cabe señalar que la consistencia interna según los objetivos de la medición es adecuada en valores entre 0.70 y $0.95 .^{1,15} \mathrm{La}$ CAT ha mostrado valores superiores a 0.95 en varias investigaciones, lo que sugiere en la CAT la existencia de redundancia o alta colinealidad entre algunas preguntas que parten de ella. Para mejorar esto se debe eliminar uno o más de los ítems que conforman la misma. ${ }^{15}$

Estudios previos no han evaluado la validez nomológica de la CAT con la correlación de Spearman. Y los hallazgos en el presente estudio ponen en cuestionamiento la eventual utilidad clínica de la CAT como indicador de calidad de vida en paciente con EPOC. Esto se corrobora con los estadísticos que mostraron que la validez discriminante de las puntuaciones en los distintos grupos según la clasificación GOLD, es pobre cuando se aplican estadísticos más robustos como la prueba de Tukey que realiza múltiples comparaciones entre los grupos; esto es similar a lo que informaron Pothirat et al., observaron que no hubo diferencia estadísticamente significativa en las puntuación de la CAT según la gradación GOLD ( $A, B, C$ y D).10

Para garantizar la validez de los instrumentos como la CAT es indispensable observar indicadores favorables en distintas poblaciones y con diferentes aproximaciones metodológicas. ${ }^{1,18}$ En el presente estudio, la estructura interna de la CAT con una única dimensión fue insatisfactoria. En el AFE el ítem 1 (tos) y el ítem 2 (flema) se comportan con un factor independiente; no obstante, el ajuste del modelo no fue adecuado como era de esperar porque para que un factor se acepte como tal debe tener por lo menos tres preguntas que miden dicha dimensión. ${ }^{28}$ No es posible comparación alguna dado que los estudios previos se conformaron con otras pruebas de validez menos robustas que las que se infiere a partir de un AFC. ${ }^{2-5}$

Es importante revisar de manera asidua el rendimiento clinométrico de los instrumentos que se usan en salud como la CAT da la inestabilidad frecuente en 
los mismos, el comportamiento cambia ampliamente según la población. Los hallazgos sugieren que es limitada la utilidad de la CAT en la evaluación clínica de la calidad de vida en pacientes con EPOC.,28 De tal suerte que las investigaciones que realicen mediciones de calidad de vida con la CAT en pacientes con EPOC, es posible que presenten una pobre validez interna y, en consecuencia, se deben interpretar las conclusiones del estudio con extrema precaución. ${ }^{29}$

El principal aporte de esta investigación es mostrar el desempeño clinimétrico de la CAT en español, informar una segunda estimación de consistencia interna y realizar el primer AFE y AFC. Asimismo, destaca la importancia de probar la confiabilidad y la validez de los instrumentos de medición en salud con múltiples aproximaciones estadísticas. ${ }^{30}$ Sin embargo, esta investigación tiene la limitación propia de los estudios clinimétricos que son difícilmente generalizables, con independencia del tipo de muestreo realizado.,15,28,30

\section{CONCLUSIONES}

Se concluye que la CAT muestra aceptable consistencia interna en pacientes con EPOC de Santa Marta, Colombia. No obstante, la estructura unidimensional originalmente propuesta presenta un ajuste muy deficiente y la validez nomológica y discriminante es pobre. Es necesario corroborar estos hallazgos en otras poblaciones para realizar las modificaciones pertinentes para las preguntas que hacen parte del instrumento.

\section{REFERENCIAS}

1. Keszei AP, Novak M, Streiner DL. Introduction to health measurement scales. J Psychosom Res 2010;68(4):319323. doi: 10.1016/j.jpsychores.2010.01.006.

2. Jones PW, Harding G, Berry P, Wiklund I, Chen WH, Kline Leidy N. Development and first validation of the COPD Assessment Test. Eur Respir J 2009;34(3):648-654. doi: 10.1183/09031936.00102509.

3. Gupta N, Pinto LM, Morogan A, Bourbeau J. The COPD assessment test: a systematic review. Eur Respir J 2014;44(4):873-884. doi: 10.1183/09031936.00025214.

4. Weldam SW, Schuurmans MJ, Liu R, Lammers JW. Evaluation of quality of life instruments for use in COPD care and research: a systematic review. Int J Nurs Stud 2013;50(5):688-707. doi: 10.1016/j.ijnurstu.2012.07.017.

5. Jones PW. The COPD Assessment Test: what have we learned over its first 5 years? Eur Respir J 2014;44(4):833834. doi: 10.1183/09031936.00125214.

6. Hwang YI, Jung KS, Lim SY, Lee YS, Kwon NH. A validation study for the Korean version of chronic obstructive pulmonary disease assessment test (CAT). Tuberc Respir Dis 2013;74(6):256-263. doi: 10.4046/ trd.2013.74.6.256.

7. Silva GP, Morano MT, Viana CM, Magalhães CB, Pereira ED. Portuguese-language version of the COPD Assessment Test: validation for use in Brazil. J Bras Pneumol 2013;39(4):402-408. doi: 10.1590/S180637132013000400002.

8. Agustí A, Fernández-Villar A, Capelastegui A, GarcíaLosa M, Velasco B, Sánchez G. Estudio de la validez de las versiones en catalán, gallego y vasco del cuestionario COPD assessment test y equivalencia con la versión en castellano. Arch Bronconeumol 2017;53(6):311-317. doi: 10.1016/j.arbres.2016.10.003.

9. Lari SM, Ghobadi H, Attaran D, Mahmoodpour A, Shadkam O, Rostami M. COPD assessment test (CAT): simple tool for evaluating quality of life of chemical warfare patients with chronic obstructive pulmonary disease. Clin Respir J 2014;8(1):116-123. doi: 10.1111/ crj.12047.

10. Pothirat C, Kiatboonsri S, Chuchottaworn C. Validation of the new COPD assessment test translated into Thai in patients with chronic obstructive pulmonary disease. BMC Pulm Med 2014;14(1):193. doi: 10.1186/1471-246614-193.

11. Tu YH, Zhang Y, Fei GH. Utility of the CAT in the therapy assessment of COPD exacerbations in China. BMC Pulm Med 2014;14(1):42. doi: 10.1186/1471-2466-14-42.

12. Dal Negro RW, Bonadiman L, Turco P. Sensitivity of the COPD assessment test (CAT questionnaire) investigated in a population of 681 consecutive patients referring to a lung clinic: the first Italian specific study. Multidiscip Respir Med 2014;9(1):15. doi: 10.1186/2049-6958-9-15.

13. Lee SD, Huang MS, Kang J, et al. The COPD assessment test (CAT) assists prediction of COPD exacerbations in high-risk patients. Respir Med 2014;108(4):600-608. doi: 10.1016/j.rmed.2013.12.014.

14. Yoshimoto D, Nakano Y, Onishi K, Hagan G, Jones P. The relationship between the COPD Assessment Test score and airflow limitation in Japan in patients aged over 40 years with a smoking history. Int J Chron Obstruct Pulmon Dis 2014;9:1357-1363. doi: 10.2147/COPD. S61265.

15. Campo-Arias A, Oviedo HC. Propiedades psicométricas de una escala: la consistencia interna. Rev Salud Pública 2008;10(5):831-839.

16. Bacchetti P. Current sample size conventions: flaws, harms, and alternatives. BMC Med 2010;8(1):17. doi: 10.1186/1741-7015-8-17.

17. Global Initiative for Chronic Obstructive Lung Disease. GOLD 2017. Global strategy for the diagnosis, management and Prevent of chronic obstructive pulmonary disease, updated 2014. Fecha de acceso: 01-05-2017. Disponible en: http://www.goldcopd.org

18. Ramada-Rodilla JM, Serra-Pujadas C, Delclós-Clanchet GL. Adaptación cultural y validación de cuestionarios de salud: revisión y recomendaciones metodológicas. Salud Publica Mex 2013;55(1):57-66. doi: http://dx.doi. org/10.1590/S0036-36342013000100009 
19. Agusti A, Soler-Cataluña JJ, Molina J, et al. Does the COPD assessment test (CAT(TM)) questionnaire produce similar results when self-or interviewer-administered? Qual Life Res 2015;24(10):2345-2354. doi: 10.1007/ s11136-015-0983-x.

20. Cronbach L. Coefficient alpha and the internal structure of test. Psychometrika 1951;16(3):297-334. doi. org/10.1007/BF02310555.

21. McDonald RP. The theoretical foundations of principal factor analysis and alpha factor analysis. Br J Math Stat Psychol 1970;23(Part 1):1-21.

22. Spearman C. Correlation calculated from faulty data. Br J Psychol 1910;3(3):271-295.

23. Bartlett MS. Test of significance in factor analysis. $\mathrm{Br} \mathrm{J}$ Psychol 1950;3(1):77-85.

24. Kaiser HF. An index of factorial simplicity. Psychometrika 1974;39(1):31-36.

25. IBM-SPSS Statistics for Windows, version 22.0. Armonk: SPSS. Inc.; 2013.

26. STATA 13.0. College Station: STATA; 2013.

27. Domínguez-Lara S, Merino-Soto C. Una modificación del coeficiente alfa de Cronbach por errores correlacionados
(Cartas al Editor). Rev Med Chile 2017;145(2):270-271.

28. Campo-Arias A, Herazo E, Oviedo HC. Análisis de factores: fundamentos para la evaluación de instrumentos de medición en salud mental. Rev Colomb Psiquiat 2012;41(3):659-671.

29. Sobrino E, Fernández PA, Molinari L, Arce S. Lectura crítica: Validez interna. Rev Arg Med Respir 2006;6(2):7783.

30. Post MW. What to do with "moderate" reliability and validity coefficients? Arch Phys Med Rehabil 2016;97(7):1051-1052. doi: 10.1016/j.apmr.2016.04.001.

\section{$\triangle$ Correspondencia:}

Dr. Adalberto Campo-Arias

Programa de Medicina, Facultad de Ciencias de la

Salud, Universidad del Magdalena.

Carrera 32 Núm. 22-08, Santa Marta,

Colombia, Código postal 470004.

Correo electrónico: acampoa@unimagdalena.edu.co.

Los autores declaran no tener conflicto de intereses. 